\title{
Voltage Collapse Mechanism Based on System Circuit and its Solution Manifolds
}

\author{
Yongqiang Liu Jie Wu \\ Dept. of E.E. \\ South China University of Technology \\ Guangzhou, P. R. China
}

\author{
Yixin Ni, S. M. IEEE Felix F. Wu, Fellow IEEE \\ Dept. of Electrical and Electronic Eng. \\ The University of Hong Kong \\ Hong Kong, P. R. China
}

\begin{abstract}
The problem of voltage collapse in the power system occurs when the load parameter exceeds some critical value. The critical operation point is known as the nose point in P-V curve. In this paper, the mechanism of voltage collapse is explained based on system circuit and solution manifolds. The corresponding mathematical model is derived. The proven theorem shows that the essential reason for voltage collapse is that the solution manifold of injection branch equations being not transversal with that of linear network equations. And the coincidence of the nontransversal of solution manifolds with voltage collapse and static bifurcation is proved.

Keywords: power system, voltage collapse, system manifold, static bifurcation.
\end{abstract}

\section{INTRUDUCTION}

The voltage collapse problem is of great concern to the electric utility industry. A.bnormal high or low voltages and voltage collapse may occur in many large interconnected power systems. There are many research outputs on voltage collapse analysis [1 4]. Venikov et al.[1] suggest a criterion for voltage stability based on steady state sensitivity analysis using a simple two-bus system. Tamura et al. $[2,3]$ analyze voltages collapse by multiple power flow solutions. Kwatny et al. investigate the voltage collapse by applying bifurcation analysis [4]. They show that the existence of bifurcation point is associated to the voltage collapse, and at this point the load voltages are infinitely sensitive to parameter variations. No matter from the viewpoint of multiple power flow solutions or bifurcation, it is essential to understand the nonlinear nature of power systems and the relationship between the nonlinear nature and voltage instability.

It is well known that power systems are very special large-scale nonlinear circuits, in which there are two different types of branches. One is the transmission lines which interconnect various buses, considered as network 'links' in our paper; and the other is the injection branches including generator branches and load branches, taken as the 'trees' of the network circuit hereafter. In this paper, the voltage collapse mechanism is investigated from the system solution manifold viewpoint based on structure-reserved nonlinear circuit. The paper reveals a significant fact that the voltage collapse phenomena corresponds to the occurrence of non-transversal between the solution manifold of injection branch equations and that of network equations. And the coincidence of the nontransversal of system solution manifolds mentioned above with voltage collapse and static bifurcation is proved.

\section{THE NONLINEAR CIRCUIT MODEL}

In this paper we assume all the state variables including loads are continuous variables. The equivalent circuit of a power system is a nonlinear circuit, and the injection branches, including generator branches and load branches, have nonlinear characteristics and are taken as the tree of the circuit; other branches i.e. transmission lines, are linear and taken as link branches in this paper.

\section{A. Load branch characteristic equations}

We assume that the load varies continuously. For a general load there exsit two groups of different variables, one group is the load circuit variable which has fast dynamics such as load current for inductive load, and the other is the load mechanical variable such as an induction motor slip which is a slow variable. Under most cases, the fast dynamics can be neglected, hence, under the orthogonal coordinates rotating at synchronous speed and denoted as $\mathrm{D}$ and $\mathrm{Q}$ axes, the load model using motor convention can be written as

$$
\begin{aligned}
& \dot{s}_{i}=\Delta P_{m i}\left(s_{i}, u_{i}\right) \\
& i_{i}=i_{i}\left(s_{i}, u_{i}\right)
\end{aligned}
$$

where $i=1, \cdots n_{L}, n_{L}$ is the total number of load buses. $i_{i}=\left[i_{D i}, i_{Q i}\right]^{T}, \quad u_{i}=\left[u_{D i}, u_{Q i}\right]^{T}$ are the current and voltage of the ith load branch respectively. $s_{i}$ is the slip or say the mechanical variable of the ith branch. $\Delta P_{m i}$ denotes the defference between input elecric power and output 
mechanical power. From the viewpoint of circuit, Eq. (2) is the ith branch characteristic equation, and the branch characteristics are controlled by the mechanicl variable $s_{i}$. Therefore the equation for the load current vector $i_{L}$ can be expressed as

$$
f_{L}\left(s, u_{L}, i_{L}\right)=i_{L}-i_{L}\left(s, u_{L}\right)=0
$$

where $u_{L}$ is the vector of the voltages of load buses.

In traditional analysis of power systems, for a load bus the real power and reactive power injecting to the bus is taken as load parameter and the bus is called a PQ-bus. In fact, Pand Q define the branch characteristics, and we have

$$
\left(\begin{array}{c}
i_{D i} \\
i_{Q i}
\end{array}\right)=\left(\begin{array}{l}
\left(P / U^{2}\right) u_{D i}+\left(Q / U^{2}\right) u_{Q i} \\
\left(P / U^{2}\right) u_{Q i}-\left(Q / U^{2}\right) u_{D i}
\end{array}\right)
$$

where $U=\sqrt{u_{D i}^{2}+u_{Q i}^{2}}$ is the magnitude of bus voltage. $\mathrm{P}$ and $\mathrm{Q}$ can be considered implicitly as functions of dynamic load slip s, therefore (4) can be in the format of (3) as well.

\section{B. Generator branch characteristic equation}

The 3rd-order mathematical model of a synchronous machine without damper windings under orthogonal coordinates rotating at synchronous speed can be derived as ${ }^{[7]:}$

$\dot{\delta}_{i}=\Delta \omega_{i}$

$\Delta \dot{\omega}_{i}=M_{i}^{-1}\left\{T_{m i}-D_{i} \Delta \omega_{i}+E_{q i}^{\prime}\left(i_{D i} \cos \delta_{i}+\right.\right.$

$\left.+i_{Q i} \sin \delta_{i}\right)-\Delta x_{d q i}\left[\left(i_{D i}^{2}-i_{Q i}^{2}\right) \sin \delta_{i} \cos \delta_{i}-\right.$

$\left.\left.-i_{D i} i_{Q i} \cos 2 \delta_{i}\right]\right\}$

$\dot{E}_{q i}^{\prime}=T_{d o i}^{\prime-1}\left[E_{f d}-E_{q i}^{\prime}-\Delta x_{d d i}\left(i_{D i} \sin \delta_{i}-i_{Q i} \cos \delta_{i}\right)\right]$

where $\Delta x_{d q i}=x_{q i}-x_{d i}^{\prime}, \Delta x_{d d i}=x_{d i}^{\prime}-x_{d i}$.

The generator branch characteristic equation using motor convention is

$$
\left(\begin{array}{c}
i_{D i} \\
i_{Q i}
\end{array}\right)=\left(\begin{array}{ll}
G_{D i} & B_{D i} \\
B_{Q i} & G_{Q i}
\end{array}\right)\left(\begin{array}{l}
u_{D i} \\
u_{Q i}
\end{array}\right)-\left(\begin{array}{c}
C_{D i} \\
C_{Q^{i}}
\end{array}\right) E_{q i}^{\prime}
$$

where $i=n_{L}+1, \cdots N, N=n_{L}+n_{G}$ is the numbers of buses in the power system (excepting swing bus), $n_{G}$ are the numbers of generators, and

$$
\begin{aligned}
G_{D i} & =\left[R_{i}+\left(x_{q i}-x_{d i}^{\prime}\right) \sin \delta_{i} \cos \delta_{i}\right] /\left(R_{i}^{2}+x_{q i} x_{d i}^{\prime}\right) \\
G_{Q i} & =\left[R_{i}-\left(x_{q i}-x_{d i}^{\prime}\right) \sin \delta_{i} \cos \delta_{i}\right] /\left(R_{i}^{2}+x_{q i} x_{d i}^{\prime}\right) \\
B_{D i} & =\left[x_{d i}^{\prime}+\left(x_{q i}-x_{d i}^{\prime}\right) \sin ^{2} \delta_{i}\right] /\left(R_{i}^{2}+x_{q i} x_{d i}^{\prime}\right) \\
B_{Q i} & =-\left[x_{d i}^{\prime}+\left(x_{q i}-x_{d i}^{\prime}\right) \cos ^{2} \delta_{i}\right] /\left(R_{i}^{2}+x_{q i} x_{d i}^{\prime}\right) \\
C_{D i} & =\left[R_{i} \cos \delta_{i}+x_{q i} \sin \delta_{i}\right] /\left(R_{i}^{2}+x_{q i} x_{d i}^{\prime}\right) \\
C_{Q i} & =\left[R_{i} \sin \delta-x_{q i} \cos \delta_{i}\right] /\left(R_{i}^{2}+x_{q i} x_{d i}^{\prime}\right)
\end{aligned}
$$

Therefore, the generator branch equations can be written as

$$
f_{G}\left(\delta, u_{G}, i_{G}\right)=i_{G}-i_{G}\left(\delta, u_{G}\right)=0
$$

where $u_{G}, i_{G}$ are the vectors of the voltages and currents of generator buses respectively.

\section{Network equations}

In the network the link branches i.e., transmission lines are linear, and the lumpedparameter line equivalent circuit model is employed. The network equations can be written in bus admittance matrix form:

$$
\left.\left(\begin{array}{cc}
Y_{n} & Y_{s} \\
\tilde{Y}_{s} & Y_{s s}
\end{array}\right) \begin{array}{c}
u \\
u_{s}
\end{array}\right)+\left(\begin{array}{c}
i \\
i_{s}
\end{array}\right)=0
$$

where $u=\left[u_{L}^{T}, u_{G}^{T}\right]^{T}$ and $i=\left[i_{L}^{T}, i_{G}^{T}\right]^{T}$ are the branch voltage and current vectors respectively; $\left(\begin{array}{ll}Y_{n} & Y_{s} \\ \tilde{Y}_{s} & Y_{s s}\end{array}\right)$ is the bus admittance matrix of the network; $u_{s}$ and $i_{s}$ are the voltage and abstracted current of the swing bus; and $u_{s}$ is known. From the first part of $\mathrm{Eq}(10)$ we can obtain the network equations

$$
\left(\begin{array}{ll}
Y_{11} & Y_{12} \\
Y_{21} & Y_{22}
\end{array}\right)\left(\begin{array}{l}
u_{L} \\
u_{G}
\end{array}\right)+\left(\begin{array}{l}
Y_{s 1} \\
Y_{s 2}
\end{array}\right) u_{s}+\left(\begin{array}{l}
i_{L} \\
i_{G}
\end{array}\right)=0
$$

or

$$
f_{N}(u, i)=Y_{n} u+Y_{s} u_{s}+i=0
$$

\section{Integrated model for power systems}

Based on the model derived above, we can see the integrated power system model consists of two sets of equations, the first set of equations is the differential equations of (1) and (5) to (7), the second set is the algebraic equations, shown in (3), (9) and (12). The dynamical behavior of variables $s, \delta$ and $E_{q}^{\prime}$ is governed by the differential equations. It is easy to see that the dynamic behavior of $s, \delta$ and $E_{q}^{\prime}$ has significant impacts on the tree branch characteristics

\section{SOLUTION MANIFOLDS AND EQUILIBRIA}

It is clear that the equilibria of the power system are determined by (3), (9), (12) and $\dot{s}=0, \dot{\delta}=0$, $\Delta \dot{\omega}=0$, and $\dot{E}_{q}^{\prime}=0$. If the space discussed is restricted on injection currents and injection voltages $(I-U)$ space, we can analyze the nature of the system solution manifolds of (3), (9) and (12) and get deep insight of the system. 
Theorem 1[5]: If $M$ is an $m$-dimensional manifold, $F: M \rightarrow R^{n}(n \leq m)$ is a smooth mapping. If $F$ has maximum rank for the subset $N=\{x \mid F(x)=0\}$, then $N$ is a regular sub-manifold of dimension $m-n$.

Combining (3), $(5 \sim 7)$ and (8), the injection branch equations take the form of

$$
\left(\begin{array}{c}
i_{L} \\
i_{G}
\end{array}\right)-\left(\begin{array}{c}
i_{L}\left(s\left(u_{L}\right), u_{L}\right) \\
i_{G}\left(\delta\left(i_{G}\right), u_{G}\right)
\end{array}\right)=0
$$

or

$$
f_{\text {inj }}\left(s\left(u_{L}\right), \delta\left(i_{G}\right), u_{L}, u_{G}, i_{L}, i_{G}\right)=0
$$

where $s\left(u_{L}\right)$ and $\delta\left(i_{G}\right)$ are solved from $\dot{s}=0$, $\dot{\delta}=0, \Delta \dot{\omega}=0$ and $\dot{E}_{q}^{\prime}=0$. It is clear the injection branch is the controlled branch, and the branch steady state characteristics are controlled by $s\left(u_{L}\right)$ and $\delta\left(i_{G}\right)$.

Theorem 2: The solution manifold of network equation (12) is a $2 N$-dimensional regular submanifold in $4 N$-dimensional injection currentnode voltage space.

Proof:

The matrix $\left(\frac{\partial f_{N}}{\partial i} \frac{\partial f_{N}}{\partial u}\right)$ has full rank. Using theorem 1 this theorem can be proved directly.

Remark: It should be pointed out that when the parameters and the structure of power systems are invariant, the solution manifold of (12) is also invariant with respect to the variation of load parameters. ( $\mathrm{P}$ or $\mathrm{Q}$ for $\mathrm{PQ}$-bus load model or output mechanical toque of the induction motor etc. are all load parameters.)

Theorem 3: If the mapping of $f_{i n j}$ is smooth, then the solution manifold of $f_{i n j}=0$ is a regular submanifold of dimension $2 \mathrm{~N}$ in $4 \mathrm{~N}$-dimensional $I$ $U$ space.

Proof:

Since the matrix

$$
\left(\begin{array}{ll}
G_{D i} & B_{D i} \\
B_{Q i} & G_{Q i}
\end{array}\right)
$$

is of full rank for any $\delta$, therefore, $\partial f_{G} / \partial u_{G}$ must be nonsingular. And also since the matrix $\partial f_{L} / \partial i_{L}=I$, therefore, injection branch equations $f_{i n j}=0$ has maximum rank. According to theorem 1 this theorem can be proved directly.

From theorems 2 and 3, we can see that under some fair conditions, the solution manifolds of injection branch equations and network equations are all regular submanifolds of injection currentnode voltage space. Let $M_{N}$ and $M_{i n j}$ be the solution manifolds of (12) and $f_{i n j}=0$ respectively, then the equilibrium point $(i, u)$ is

$$
(i, u)=M_{i n j} \cap M_{N}
$$

which means that when the conditions of theorem 2 are satisfied, the equilibrium point of the nonlinear circuit associated to the power system is the intersection of two regular submanifolds $M_{i n j}$ and $M_{N}$ in $I-U$ space.

\section{VOLTAGE COLLAPSE IN A SIMPLE SYSTEM}

Now we apply the conclusion to study the voltage collapse mechanism perceptually. As an example, a simple single machine - single load power system is used with its equivalent circuit shown in Fig.1.

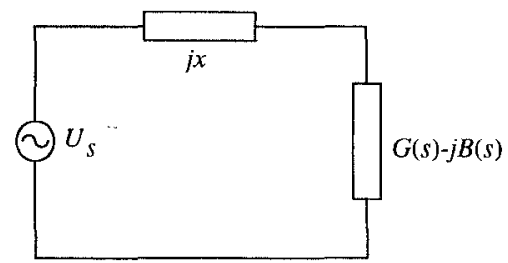

Fig. 1 Equivalent circuit for the simple system

In Fig. 1, $x$ is the inductance of the transmission line; $G(s)-j B(s)$, the equivalent admittance of the induction motor; $s$, the slip of the motor, and

$$
\begin{aligned}
& G(s)=\left(r_{1}+r_{2}^{\prime} / s\right) /\left[\left(r_{1}+r_{2}^{\prime} / s\right)^{2}+X^{2}\right] \\
& B(s)=X /\left[\left(r_{1}+r_{2}^{\prime} / s\right)^{2}+X^{2}\right] \\
& s=r_{2}^{\prime} /\left[\frac{U^{2}}{2 P_{m}}+\sqrt{\left.\left(\frac{U^{2}}{2 P_{m}}\right)^{2}-X^{2}-r_{1}\right]}\right.
\end{aligned}
$$

where $r_{1}, r_{2}^{\prime}$ are the resistance of stator and rotor windings respectively viewed from stator, $P_{m}$ is the output mechanical power of the induction motor load. $X$ is the summation of inductance of stator and rotor windings. $U$ is the voltage of the load bus. Substituting (17) into (15) and (16), we can have $G(s)-j B(s)=g(U)-j b(U)$. Therefore the load injection branch equation is:

$$
I-U \sqrt{g^{2}(U)+b^{2}(U)}=0
$$

and the network (line) equation is:

$$
U_{S}^{2}=(U+I x \sin \varphi)^{2}+(I x \cos \varphi)^{2}
$$


where, $\varphi=\tan ^{-1}\left[X /\left(\frac{U^{2}}{2 P_{m}}+\sqrt{\left.\left(\frac{U^{2}}{2 P_{m}}\right)^{2}-X^{2}\right)}\right]\right.$.

Fig.2 shows the solution curves of (18) (see curve 1) and (19) (see curve 2). When system operate at normal conditions, the corresponding curve 1 intersects the relevant curve 2 at points $b$ and $a$, point $b$ corresponds to normal voltage level and stable load flow solution, while point $a$ is an abnormal operation point. When $P_{m}$ increases to some critical value $P_{m c}$, the curves 1 and 2 change to 1' and 2' respectively, and point $a$ and point $b$ coincide at point $c$, at which point the solution manifold 1' of (18) is tangent to the solution manifold 2' of (19). It is clear that the geometrical structure of the solution manifolds of (18) and (19) is unstable when $P_{m}=P_{m c}$. Once $P_{m}>P_{m c}$, the system will lose the equilibrium point near point $c$, i.e., voltage collapse phenomena occur.

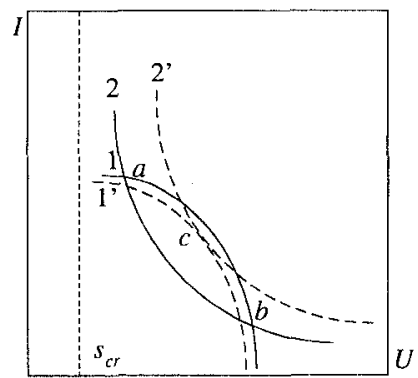

Fig.2 Schematic diagram for voltage collapse in the simple system

\section{VOLTAGE COLLAPSE IN GENERAL POWER SYSTEMS}

From above analysis, we can see that voltage collapse is dependent on the solution manifolds of the injection branch equations and the network equations of power systems. However, both the injection equations and network equations usually have very high dimensions in a complex power system. Hence, how to explain the relation between voltage collapse and the nonlinear characteristics of nonlinear circuits of the studied system is an issue we have to address. In order to make further analysis on voltage stability, the following definition and lemma are given first.

Definition 1[6]: Let $S$ and $L$ be two sub-manifolds of $N$, if $p \in S \cap L$, and

$$
T_{p} S+T_{p} L=T_{p} N
$$

then $S$ and $L$ at point $p$ are transversal; otherwise they are not transversal at point $p$. Here $T_{p} S, T_{p} L$ and $T_{p} N$ express tangent spaces of $S, L$ and $N$ respectively.

Let $f(x, y)$ and $g(x, y)$ be two vector fields with $C^{r}, r \geq 1, \quad x \in R^{n}, \quad y \in R^{m}$. Consider following two sets of equations

$$
\begin{aligned}
& f(x, y)=0 \\
& g(x, y)=0
\end{aligned}
$$

Assumption 1: The following two matrices satisfy

$$
\begin{aligned}
& \operatorname{rank}\left(\frac{\partial f}{\partial x} \quad \frac{\partial f}{\partial y}\right)=n \\
& \operatorname{rank}\left(\frac{\partial g}{\partial x} \quad \frac{\partial g}{\partial y}\right)=m \text {. }
\end{aligned}
$$

It is clear that the solution manifolds of (21) and (22) are two regular submanifolds from theorem 1.

Lemma 1: According to assumption 1, the necessary and sufficient conditions of the solution manifolds of (21) and (22) being transversal at $\left(x_{p}, y_{p}\right)$ in $R^{n+m}$ are

$$
\operatorname{rank}\left(\begin{array}{ll}
\frac{\partial f}{\partial x} & \frac{\partial f}{\partial y} \\
\frac{\partial g}{\partial x} & \frac{\partial g}{\partial y}
\end{array}\right)_{\left(x_{p}, y_{p}\right)}=n+m
$$

Proof:

Using definition 1, the lemma can be proved.

Apply above mathematical theory to power systems, we can obtain the following theorems.

Theorem 4: Assume the power system model, including (5), (6), (7), (12) and (13), to be smooth. If the solution manifold of (12) and the solution manifold of (13) are not transversal at operation point $\left(i_{p}, u_{p}\right) \in M_{i n j} \cap M_{N}$ for equilibrium point $\delta=\delta\left(i_{p}\right)$ and $s=s\left(u_{p}\right)$, i.e.,

$$
\tilde{J}=\left(\begin{array}{cc}
I_{N \times N} & Y_{n} \\
\frac{\partial f_{i n j}}{\partial i}+\frac{\partial f_{i n j}}{\partial \delta} \frac{\partial \delta}{\partial i} & \frac{\partial f_{i n j}}{\partial u}+\frac{\partial f_{i n j}}{\partial s} \frac{\partial s}{\partial u}
\end{array}\right)_{\left(i_{p}, u_{p}\right)}
$$

is singular, then under a small perturbation the power system will lose the equilibrium point near $\left(i_{p}, u_{p}\right)$, i.e., voltage collapse phenomena occur.

Proof:

Using lemma 1, this theorem can be proved.

Remark: Theorem 4 shows that the two solutionmanifolds $M_{i n j}$ and $M_{N}$ being not transversal to each other in the injection branch current and node 
voltage spanned space causes voltage collapse. In other words, injection branch characteristics have great influences on the transversal of $M_{i n j}$ and $M_{N}$. It also means that the nonlinear nature of injection branches dominates the voltage stability.

\section{THE RELATION BETWEEN THE TRANSVERSAL OF $M_{i n j}$ AND $M_{N}$ AND STATIC BIFURCATION}

In [4] Kwatny et al. analyzed static bifurcation in power systems, and studied the relationship between static bifurcation phenomena and voltage collapse. In this section authors study the relation between static bifurcation and the transversal of $M_{i n j}$ and $M_{N}$.

From above analysis, the power system can be modeled as

$$
\begin{aligned}
\dot{x} & =f(x, u) \\
0 & =f_{N}(i, u) \\
0 & =f_{i n j}(x, i, u)
\end{aligned}
$$

where $x^{T}=\left[\delta^{T}, \Delta \omega^{T}, E_{q}^{\prime T}, s^{T}\right]$ denotes the state variables.

Assumption 2: That the Jacobian matrix is singular corresponds to the static state bifurcation.

Assumption 3: The matrix $\partial f / \partial x$ is nonsingular for the interested equilibrium point.

Above assumption 3 is usually reasonable, since that $\partial f / \partial x$ is singular means the system is at critical operation point for the voltage of generator being a constant. It is easy to see that in fact the critical point of interest occurs prior to $\operatorname{det}[\partial f / \partial x]=0$. Hence, assumption 3 is fair.

Theorem 5: If the solution-manifolds $M_{i n j}$ and $M_{N}$ are not transversal at $\left(i_{p}, u_{p}\right) \in M_{i n j} \cap M_{N}$ for equilibrium point $\delta=\delta\left(i_{p}\right)$ and $s=s\left(u_{p}\right)$, then according to assumptions 2 and $3,\left(i_{p}, u_{p}\right)$ must be corresponding to a static bifurcation point. Proof:

When the static bifurcation occurs at point $\left(i_{p}, u_{p}\right)$ in the power system (see (25-27)), the following matrix

$$
J=\left(\begin{array}{ccc}
\frac{\partial f}{\partial x} & \frac{\partial f}{\partial i} & \frac{\partial f}{\partial u} \\
0 & I & Y_{n} \\
\frac{\partial f_{i n j}}{\partial x} & \frac{\partial f_{i n j}}{\partial i} & \frac{\partial f_{i n j}}{\partial u}
\end{array}\right)_{\left(i_{p}, u_{p}\right)}
$$

must be singular. By Schur's formula

$$
\begin{aligned}
& \operatorname{det} J=\left(\begin{array}{cc}
I & Y_{n} \\
\frac{\partial f_{i n j}}{\partial i} & \frac{\partial f_{i n j}}{\partial u}
\end{array}\right)-\left(\begin{array}{c}
0 \\
\frac{\partial f_{i n j}}{\partial i}
\end{array}\right)\left(\frac{\partial f}{\partial x}\right)^{-1}\left(\frac{\partial f}{\partial i} \frac{\partial f}{\partial u}\right) \\
& =\left(\begin{array}{cc}
I \\
\frac{\partial f_{i n j}}{\partial i}-\frac{\partial f_{i n j}}{\partial x}\left(\frac{\partial f}{\partial x}\right)^{-1} \frac{\partial f}{\partial i} & \frac{\partial f_{i n j}}{\partial u}-\frac{\partial f_{i n j}}{\partial x}\left(\frac{\partial f}{\partial x}\right)^{-1} \frac{\partial f}{\partial u}
\end{array}\right)(28)
\end{aligned}
$$

From implicit function theorem, we know at the equilibrium point

$$
\begin{aligned}
\left(\begin{array}{cc}
\frac{\partial \delta}{\partial i} & 0 \\
0 & \frac{\partial s}{\partial u}
\end{array}\right) & =-\left(\frac{\partial f}{\partial x}\right)^{-1}\left(\begin{array}{ll}
\frac{\partial f}{\partial i} & \frac{\partial f}{\partial u}
\end{array}\right)= \\
& =-\left(\left(\frac{\partial f}{\partial x}\right)^{-1}\left(\frac{\partial f}{\partial i}\right)\left(\frac{\partial f}{\partial x}\right)^{-1}\left(\frac{\partial f}{\partial u}\right)\right)
\end{aligned}
$$

Then we have

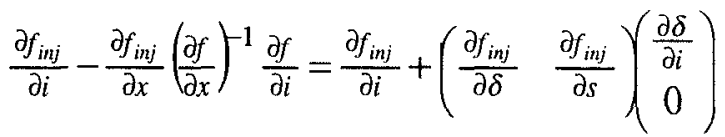

$$
\begin{aligned}
& =\frac{\partial f_{i n j}}{\partial i}+\frac{\partial f_{i n j}}{\partial \delta} \frac{\partial \delta}{\partial i} \\
& \frac{\partial f_{i n j}}{\partial u}-\frac{\partial f_{i n j}}{\partial x}\left(\frac{\partial f}{\partial x}\right)^{-1} \frac{\partial f}{\partial u}=\frac{\partial f_{i n j}}{\partial u}+\left(\begin{array}{ll}
\frac{\partial f_{i n j}}{\partial \delta} & \frac{\partial f_{i n j}}{\partial s}
\end{array}\right)\left(\begin{array}{c}
0 \\
\frac{\partial s}{\partial u}
\end{array}\right) \\
& =\frac{\partial f_{i n j}}{\partial u}+\frac{\partial f_{i n j}}{\partial s} \frac{\partial s}{\partial u}
\end{aligned}
$$

Substituting (30) and (31) into (28), we can see $\operatorname{det}[J]=0$ means that $\tilde{J}$ is also a singular matrix. Therefore, the point at which the solutionmanifolds of $M_{i n j}$ and $M_{N}$ are not transversal to each other is corresponding to the saddle-node bifurcation point in $I-U$ space.

\section{CONCLUSIONS}

By above analysis, main conclusions of this paper can be drawn as follows.

- Complete power systems can be described in state variable space with slow dynamics and circuit variables (injection branch current and voltage).

- The solution manifold $M_{i n j}$ of injection branch equations and the solution manifold $M_{N}$ of linear network equation are all regular submanifolds of the space spanned by 
the injection currents and corresponding voltages.

- The occurrence of solution-manifolds of $M_{i n j}$ and $M_{N}$ being not transversal to each other means the loss of the equilibrium point near $\left(i_{p}, u_{p}\right)$, i.e., the happening of voltage collapse phenomenon.

- The critical intersection point of solution manifolds $M_{i n j}$ and $M_{N}$ when they are not transversal is corresponding to the static bifurcation point.

\section{ACKNOWLEDGMENTS}

This research is supported by National Key Basic Research Special Fund No. G1998020307, to whom sincere acknowledgment is expressed.

\section{REFERENCE}

[1] V.A.Venikov and M.N.Rozonov, "The stability of a load" Izv. Akad. Nauk SSSR (Energetika i Arto matica), vol.3 1961, pp. 121-125.

[2] Y.Tamura, H. Mori and S.Iwamoto, "Relationship between voltage instability and multiple load flow solutions in electric power systems", IEEE Trans. on Power Apparatus and Systems, vol. PAS-102, May 1983, pp.1115-1125.

[3] Y.Tamura, K.Sakamoto, and Y.Tayama, "Voltage instability proximity index (VIPI) based on multiple load flow solutions in ill-conditions power systems", Proc. IEEE Conference on Decision and Control, Austin, Texas December 1988, pp. 2114- 2119.

[4] H. G. Kwantny, A. K. Pasrija, and L. Y. Bahar, "Loss of steady state stability and voltage collapse in electric power systems", Proc. IEEE Conference on Decision and Control, Ft. Lauderdale, FL. Dec.1985, pp.804-811.

[5] Li Ji-Bin, Zhao Xiao-Hua, Liu Zheng -Rong, "Generalized Hamilton system theory and its application", Science Press of China, Beijing, 1994. (in Chinese)

[6] Yang Wan-Nian, " Differential manifolds and its application" Chongqing: Chongqing University Press. 1992. (in Chinese)

[7] P. Kendur, "Power system stability and control", McGraw Hill, 1994

\section{BIOGRAPHY}

Yongqiang Liu received his B. Eng. from Huazhong University of Science and Technology P. R. China in 1982, and received his Dr. Eng. South China University of Technology P. R. China in 1999, both is in Electrical Engineering Department. His research interest is in system science, nonlinear control and power system stability analysis and control.

Jie $W u$ received his B.Eng. from Dept. of $\mathbb{E}$. E., Harbin Institute of Technology in 1961. His interest is automatic control theory, including adaptive control, AI, nonlinear control and power systems control. He is a professor of Dept. of E. E., South China University of Technology.

Yixin $\mathrm{Ni}$ received her B. Eng., M. Eng., and Dr. Eng. from Electrical Engineering Department, Tsinghua University, P. R. China in 1968,1981 and 1983 respectively. Her research interest is in power system modeling, simulation, stability and control, and power electronics applications in power systems. She was a professor of Tsinghua University and is now with the University of Hong Kong.

Felix $F$. Wu joined the University of Hong Kong as Chair professor of Electrical Engineering in September 1995 and is former Pro-vice-chancellor of HKU. Prior to that, he was Professor and Vice Chairman, Department of Electrical Engineering and Computer Sciences, University of California, Berkeley, the same Institute where he received his Ph.D. degree. His research interests are in power system planning and operation, including economics and reliability in system planning, real-time security assessment, and design of energy management systems and distribution automation. Recently he has been involved in the design of industry restructuring and electricity pricing. 